\title{
The Effect of Enhanced Multislice Spiral Computed Tomography Scan in Nursing
}

\author{
Yan Wang, Binyan Qian, and Jidong Peng $\mathbb{B}$ \\ Department of Radiology, Ganzhou People's Hospital, 341000 Jiangxi, China \\ Correspondence should be addressed to Jidong Peng; jidongpeng2021@163.com
}

Received 21 October 2021; Revised 12 December 2021; Accepted 25 January 2022; Published 16 February 2022

Academic Editor: Kelvin Wong

Copyright (C) 2022 Yan Wang et al. This is an open access article distributed under the Creative Commons Attribution License, which permits unrestricted use, distribution, and reproduction in any medium, provided the original work is properly cited.

\begin{abstract}
Purpose. Side effects such as local pain and tissue swelling will affect the effect of enhanced multislice spiral computed tomography (CT) scan. The aim of this study was to explore the effect of imaging nursing in enhanced CT scan for heart patients. Method. We selected 86 heart patients to receive enhanced multislice spiral CT scan as the research objects and randomly divided them into the test group and the control group. Imaging nursing and traditional nursing intervention was taken to compare the nursing effects of the two groups. Results. The incidence of unpleasant mood in the test group was 9.3\%, which was significantly lower than that in the control group. The nursing satisfaction of the test group was $97.7 \%$, which was much higher than the $79.1 \%$ of the control group. At the same time, the visual analogue scale (VAS) score of the test group and the incidence of contrast agent extravasation were significantly lower than those of the control group. Conclusion. The use of imaging nursing for heart patients with enhanced multislice spiral CT scan can reduce the patient's unhealthy mood and improve nursing satisfaction, and the nursing effect is ideal.
\end{abstract}

\section{Introduction}

Multislice spiral computed tomography- (CT-) enhanced scanning is commonly used in clinical practice [1-4], which belongs to computer scanning technology. The clinical advantages [5-7] include clear images, short scanning time, and qualitative and positioning diagnoses and are widely used in clinical examinations. In CT-enhanced scanning, water-soluble contrast agents need to be injected intravenously.

We commonly used contrast agents which include nonionic contrast agents and ionic contrast agents. The former has relatively few adverse reactions, but the price is higher, and the latter is cheap but easy adverse reactions may be taken seriously during the examination. Thus, corresponding nursing measures should be applied to the heart patients.

The clinically enhanced CT scan of heart patients requires intravenous infusion of water-soluble contrast agents to ensure that the X-ray absorption rate of the patient's diseased tissues is increased [8-10], which can increase the density of normal tissues and diseased tissues and the difference in show efficiency. Multislice spiral CT enhanced scanning on heart patients can easily lead to related leakage of the contrast agent [11-16], which will lead to low patient compliance and decreased satisfaction.

The contrast-enhanced imaging examination is mainly based on a plain scan $[17,18]$, and then, the diseased tissue can be qualitatively diagnosed through the difference of contrast agent density. Contrast-enhanced scanning requires high-pressure injection when the contrast agent is injected. However, due to individual differences in heart patients, some heart patients will ooze out after the injection of the contrast agent. The contrast agent itself will have a certain impact on the local skin tissue of the patient, mainly local pain and tissue swelling.

With the rapid development of enhanced imaging examinations, enhanced imaging examinations have been widely used in medical imaging diagnosis and differential diagnosis, which can improve the detection rate of lesions and the accuracy of diagnosis. However, if the exudation is severe, serious consequences such as tissue necrosis and limb dysfunction may occur. Therefore, predictive nursing interven- 


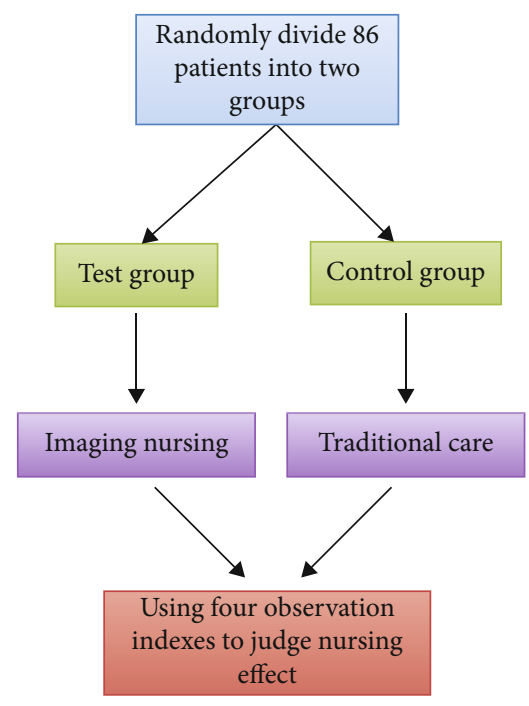

Figure 1: The specific structure of the control experiment.

tion is particularly important.

As the success or failure of contrast agent injection for enhanced imaging examination will directly affect the density identification of diseased tissues and normal tissues, the care before and after the enhanced imaging examination is one of the important links to ensure accurate and effective image information. Therefore, the aim of this study was to explore the effect of imaging nursing in an enhanced CT scan for heart patients.

\section{Materials and Methods}

2.1. General Information. According to the established control experiment structure, this paper studies the imaging nursing effect of enhanced scanning with multislice spiral CT. The experimental structure is shown in Figure 1.

The 86 heart patients with enhanced imaging examination in our hospital were randomly divided into two groups. The number of cases in each group is 43 . The age of the test group was 20-73 years, the average age was $44.9 \pm 7.3$ years, and the male to female ratio was $24: 19$. The control group was 21 to 70 years old, with an average age of $44.0 \pm 6.4$ years old, and the ratio of males to females was $20: 23$. The comparison of the two groups of samples showed no significant difference $(t=0.608, P=0.545)$. The specific situation is shown in Figure 2.

\subsection{Method}

2.2.1. Control Group. A total of 43 cases were treated with traditional care. After the scan, the heart patients were evaluated psychologically. Traditional nursing includes basic nursing, treatment and medication, rehabilitation guidance, health education, psychological nursing, and other nursing work.

2.2.2. Test Group. A total of 43 cases were treated with imaging care. The specific steps are as follows $[19,20]$.
The first step is to measure and record the patient's blood pressure, height, weight, etc. before the examination, which helps to choose the contrast agent and determine the amount of contrast agent. The nurse should carefully read the examination application form and patiently ask the patient's condition and allergy history to determine whether it is suitable for CT-enhanced examination. If the patient needs an abdominal examination, fasting can avoid intestinal substances affecting the examination results. Barium meal examinations have been carried out recently, and CT-enhancement examinations can only be carried out after 1 week. Before the examination, check whether the patient has residual barium meal. If residual, use diatrizoate meglumine at a dose of $1000 \mathrm{ml} 2$ hours before the examination. And then, we use $500 \mathrm{ml}$ to exclude heart patients with allergies and asthma from other substances, exclude heart patients with contraindications, and strictly control the examination of high-risk heart patients.

In the second step, the patient may have emotions such as fear and anxiety due to the examination. Before the examination, the nurse should guide the patient's psychology, explain the knowledge of the contrast agent, and encourage the patient to actively cooperate with the examination.

The third step is to strictly abide by the relevant operating regulations during the scan to avoid liquid leakage. The nurse should make a reasonable choice of the injection position according to the actual situation. During the puncture process, strictly perform aseptic operations and select the venous lumens of the extremities. After completing the puncture and doing a good job of drug injection, place the puncture arm to a comfortable position and instruct the patient to rest for 0.5 to 1 hour and relax. If it is painful, the nurse should inject analgesic drugs according to the patient's pain.

In the fourth step, after the scan, the nurse should assist the patient to get out of bed and instruct the patient to drink plenty of water, which can accelerate the excretion of drugs. All kinds of first aid supplies are prepared in the CTenhanced scanning room to actively respond to unforeseen adverse reactions. If the patient is mildly allergic and needs to drink plenty of water and rest and if the patient is moderately allergic, the intravenous channel needs to be established quickly and injected with $10 \mathrm{mg}$ dexamethasone, if necessary, $0.5 \sim 1 \mathrm{mg}$ epinephrine, and rescue preparations will be made. Well, if the patient is severely allergic, he should be rescued immediately, the emergency department shall be notified, and the relevant cooperation work will be done to treat the patient in the shortest time.

\subsection{Observation Indicators}

2.3.1. Incidence of Unpleasant Mood. The negative emotions we studied include tension, uneasiness, and anxiety.

2.3.2. Nursing Satisfaction. Questionnaires were distributed to heart patients to count nursing satisfaction. The survey results were divided into 3 levels: very satisfied $(\mathrm{V})$, generally satisfied $(\mathrm{G})$, and not satisfied $(\mathrm{N})$. The calculation formula 


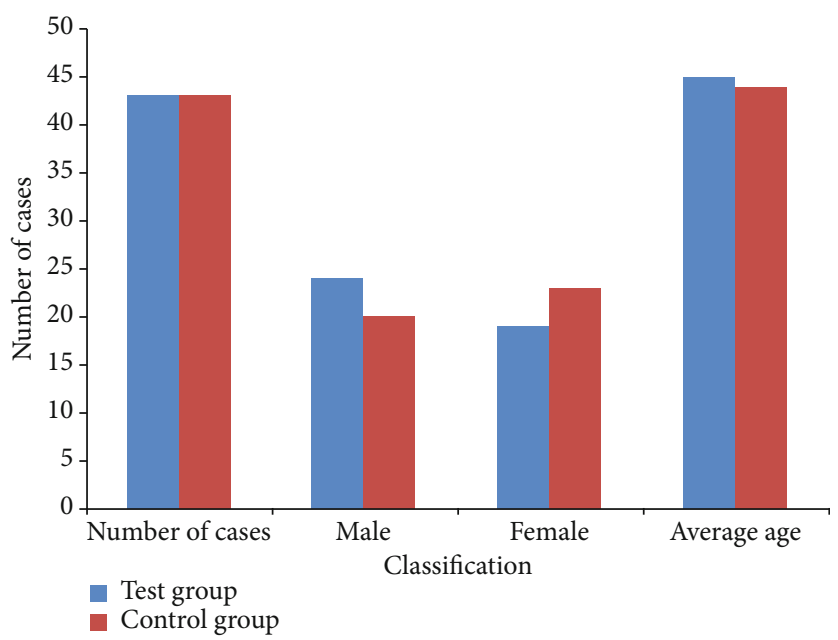

FIGURE 2: Details of sample data.

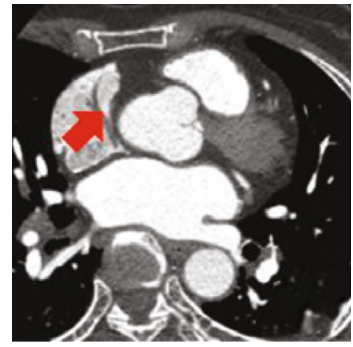

(a)

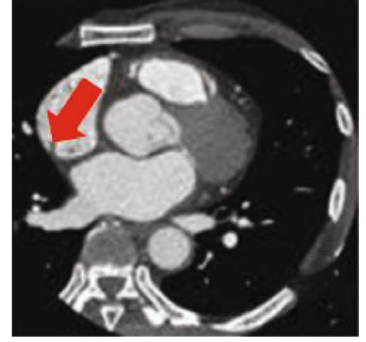

(b)

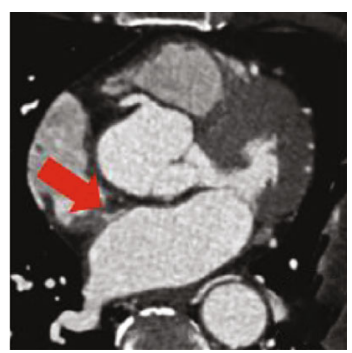

(c)

Figure 3: Multislice spiral CT image of a heart disease patient.

of nursing satisfaction is as follows:

$$
N=\frac{V+G}{T} \times 100 \%
$$

In Equation (1), $N$ represents nursing satisfaction, $V$ represents very satisfactory, $G$ represents general satisfaction, and $T$ represents the total number of cases.

2.3.3. Visual Analogue Scoring Method. This paper uses the visual analogue scale (VAS) [21-24] to assess the patient's pain level. The total score is 10 points. The higher the VAS score is, the more severe the pain.

2.3.4. Incidence of Contrast Media Extravasation. The calculation of the incidence of contrast agent extravasation is actually the ratio of the number of cases of contrast agent extravasation to the total number of cases.
2.4. Statistical Processing. SPSS 26.0 software processes the data, counts for chi-square statistics, and measures data for $t$ test. $P<0.05$ indicates that the difference is meaningful.

\section{Results}

3.1. CT Images. Figure 3 shows part of the multislice spiral CT images of 86 heart patients with heart disease. The red arrow points to the part of the heart disease. The patient can be cared for more accurately through imaging. Imaging nursing and traditional nursing intervention is applied to examine the difference in treatment and diagnosis.

3.2. Comparison of Incidence of Unpleasant Mood. The incidence of unpleasant mood in the test group was significantly lower; compared with the control group, the difference was significant $(P<0.05)$. The specific situation of the 


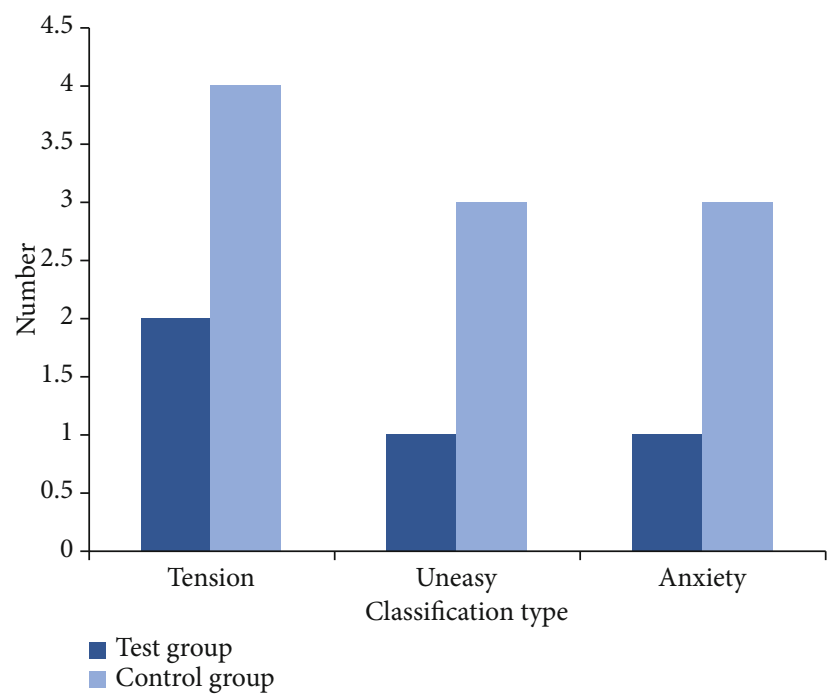

FIgURE 4: The specific situation of unpleasant emotions.

occurrence of unpleasant mood is shown in Figure 4, and the incidence of unpleasant mood and the results of statistical processing are shown in Table 1.

3.3. Comparison of Nursing Satisfaction. The nursing satisfaction of heart patients in the test group was significantly improved compared with the control group, and the difference was significant $(P=0.007)$. The specific situation of nursing satisfaction is shown in Figure 5, and the nursing satisfaction and statistical processing results are shown in Table 2.

3.4. Comparison of VAS Score. The VAS pain scores of the test group and the control group are shown in Table 3. The scores of the test group are significantly lower than those of the control group, which proves that the nursing effect of the multislice spiral CT-enhanced scanning is more effective than traditional nursing.

3.5. Comparison of Incidence of Contrast Agent Extravasation. It can be seen from Figure 6 that the incidence of contrast agent extravasation in the test group is lower than that in the control group $(P=0.042)$, and imaging nursing has a great advantage in contrast agent extravasation.

\section{Discussion}

In the process of enhanced scanning with multislice spiral $\mathrm{CT}$, heart patients need to be injected intravenously with a water-soluble organic iodine contrast agent. The X-ray absorption rate of the diseased tissue is increased. Compared with the normal tissue, the difference is obvious. The display
TABLE 1: Comparison of incidence of unpleasant mood.

\begin{tabular}{lcc}
\hline Type & Test group & Control group \\
\hline Incidence of unpleasant mood & $9.3 \%$ & $23.3 \%$ \\
$\chi^{2}$ value & & 3.071 \\
$P$ value & \multicolumn{2}{c}{0.039} \\
\hline
\end{tabular}

rate of the disease is greatly improved. The contrast is significantly enhanced.

However, due to the invasiveness of the examination and the use of contrast agents and because the heart patients do not understand the relevant knowledge, they are prone to different levels of unhealthy emotions, and patient care should be strengthened. During the nursing process, nurses should pay attention to the psychological fluctuations of heart patients, do a good job of diversion, actively implement various nursing countermeasures, prompt heart patients to actively cooperate with the examination, deepen heart patients' understanding of relevant knowledge, and prompt heart patients to successfully complete the examination [25-27]. Heart patients are provided with symptomatic treatment, which will ultimately significantly improve patient treatment effects.

Clinical studies have shown that the accuracy of CTenhanced scanning will be directly affected by the sharpness of the image. In addition to the scanning technology, the image quality is also related to the patient's mental state. Therefore, before, during, and after the enhanced multislice spiral CT scan of the patient, individualized nursing intervention should be taken to help the patient relax and maintain a good emotional state, to pay attention to observe 


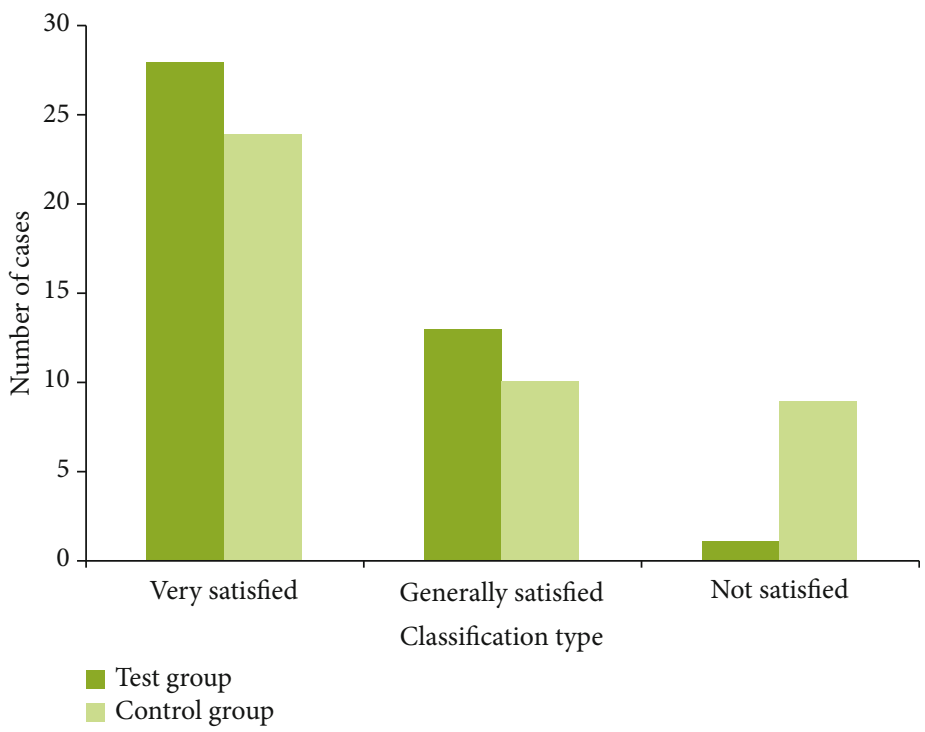

FIGURE 5: Details of nursing satisfaction.

TABLE 2: Comparison of nursing satisfaction.

\begin{tabular}{lccc}
\hline Type & Test group & \multicolumn{1}{c}{ Control group } \\
\hline Nursing satisfaction & $97.7 \%$ & & $79.1 \%$ \\
$\chi^{2}$ value & & 7.242 & \\
$P$ value & & 0.007 & \\
\hline
\end{tabular}

TABLE 3: VAS pain score of test group and control group.

\begin{tabular}{lc}
\hline Group & VAS score \\
\hline Test group & $1.2 \pm 0.5$ \\
Control group & $3.0 \pm 0.8$ \\
$\chi^{2}$ value & 12.512 \\
$P$ value & $<0.001$ \\
\hline
\end{tabular}

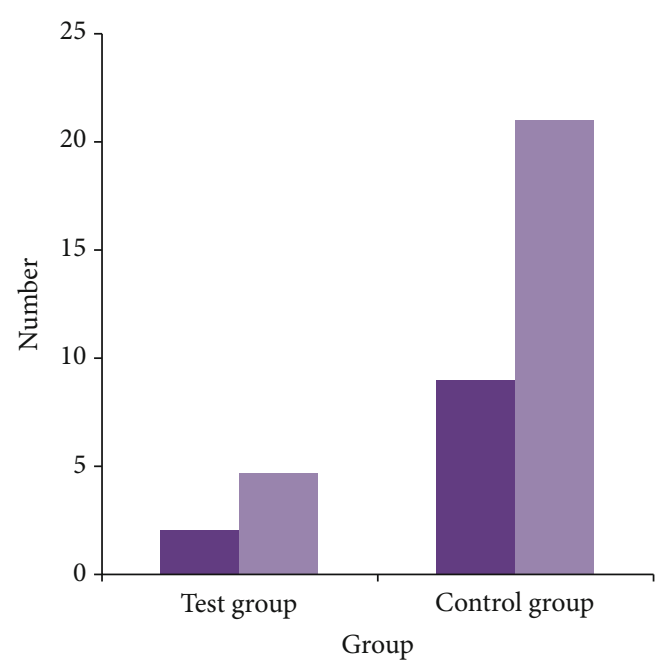

Number of cases of contrast agent extravasation Incidence of contrast agent extravasation

FIGURE 6: Comparison of the incidence of contrast agent extravasation. whether there are adverse reactions, and to do a good job of coping. The purpose of nursing is to improve the clarity of scanned images and patient satisfaction. For prediction of the optimized nursing effect, a deep learning platform [28] can be utilized to enhance the nursing intervention.

\section{Conclusion}

According to the experiments in this paper, the incidence of unpleasant emotions in the test group was significantly lower, and the data comparison between the groups was $<0.05$. The results suggest that the clinical application value of imaging nursing is higher and can be referred to clinically. In addition, the VAS pain score of the test group was lower than that of the control group, and the incidence of contrast agent extravasation was also significantly lower than that of the control group.

In summary, multislice spiral CT enhanced scan heart patients have an ideal effect and high feasibility. It can significantly alleviate heart patients' unpleasant mood and reduce the incidence of contrast agent leakage. As such, our study is clinically worth promoting.

\section{Data Availability}

The data used to support the findings of this study are available from the corresponding author upon request.

\section{Conflicts of Interest}

The authors declare that they have no conflicts of interest.

\section{References}

[1] F. Cademartiri, G. Luccichenti, and A. V. Lugt, "Sixteen-row multislice computed tomography: basic concepts, protocols, and enhanced clinical applications," Seminars in Ultrasound CT and MRI, vol. 25, no. 1, pp. 2-16, 2004. 
[2] H. Bruder, T. Flohr, and S. Schaller, "Imaging method for a multi-slice spiral CT scan with 3D reconstruction, and a computed tomography unit for carrying out this method," vol. 21, no. 3, pp. 75-79, 2004.

[3] Y. Jin-Gang, P. Wei-Bin, and Y. Rui-Gen, "Multi-slice spiral CT single-phase enhanced scanning in the pancreas," Chinese Journal of Medical Imaging Technology, vol. 22, no. 9, pp. 1380-1382, 2006.

[4] H. Scheffel, P. Stolzmann, M. J. Wilhelm et al., "Conventional radiography and computed tomography of cardiac assist devices," European Radiology, vol. 14, no. 5, pp. 186-190, 2009.

[5] A. S. Lowe and C. L. Kay, "Recent developments in CT: a review of the clinical applications and advantages of multidetector computed tomography," Imaging, vol. 18, no. 2, pp. 62-67, 2006.

[6] S. Kappler, T. Hannemann, and E. Kraft, "First results from a hybrid prototype CT scanner for exploring benefits of quantum-counting in clinical CT," vol. 57, no. 9, pp. 831383130, 2012.

[7] T. Masaryk, R. Kolonick, T. Painter, and D. B. Weinreb, “The economic and clinical benefits of portable head/neck CT imaging in the intensive care unit," Radiology Management, vol. 30, no. 2, pp. 50-54, 2008.

[8] C. Y. Shu, L. H. Gan, C. R. Wang, X. L. Pei, and H. B. Han, "Synthesis and characterization of a new water-soluble endohedral metallofullerene for MRI contrast agents," Carbon, vol. 44, no. 3, pp. 496-500, 2006.

[9] L. Nebuloni, G. A. Kuhn, and R. Müller, "A comparative analysis of water-soluble and blood-pool contrast agents for in vivo vascular imaging with micro-CT," Academic Radiology, vol. 20 , no. 10 , pp. 1247-1255, 2013.

[10] F. L. Weitl, M. Sovak, T. M. Williams, and J. H. Lang, "Studies in the design of $\mathrm{x}$-ray contrast agents. Synthesis, hydrophobicity, and solubility of some iodoresorcyl bis(beta-glucosides)," Journal of Medicinal Chemistry, vol. 19, no. 12, pp. 1359$1362,1976$.

[11] M. Hamon, G. G. L. Biondi-Zoccai, P. Malagutti et al., "Diagnostic performance of multislice spiral computed tomography of coronary arteries as compared with conventional invasive coronary angiography: a meta-analysis," Journal of the American College of Cardiology, vol. 48, no. 9, pp. 1896-1910, 2006.

[12] S. Achenbach, S. Ulzheimer, and U. Baum, "Noninvasive coronary angiography by retrospectively ECG-gated multislice spiral CT," ACC Current Journal Review, vol. 10, no. 3, pp. 44-44, 2001.

[13] M. Kachelrie, S. Ulzheimer, and W. A. Kalender, "ECG-correlated image reconstruction from subsecond multi-slice spiral CT scans of the heart," Medical Physics, vol. 27, no. 8, pp. 1881-1902, 2000.

[14] M. Vembar, M. J. Garcia, D. J. Heuscher et al., “A dynamic approach to identifying desired physiological phases for cardiac imaging using multislice spiral CT," Medical Physics, vol. 30, no. 7, pp. 1683-1693, 2003.

[15] S. Schaller and T. Flohr, "Spiral interpolation algorithm for multislice spiral CT. I. Theory," Medical Imaging IEEE Transactions on, vol. 19, no. 9, pp. 822-834, 2000.

[16] W. A. Kalender, T. Flohr, S. Schaller, J. Krause, and T. Fuchs, "Spiral interpolation algorithms for multislice spiral CT-part II: measurement and evaluation of slice sensitivity profiles and noise at a clinical multislice system," IEEE Transactions on Medical Imaging, vol. 19, no. 9, pp. 835-847, 2000.
[17] L. Bin, G. Fengling, and S. Chuan, "Analysis of the application value of plain CT scan and contrast-enhanced scan in the diagnosis of hypopharyngeal carcinoma and clinical manifestation of its adjacent structure," The Practical Journal of Cancer, vol. 86, no. 5, pp. 543-548, 2018.

[18] M. B. I. Lobbes, U. C. Lalji, P. J. Nelemans et al., "The quality of tumor size assessment by contrast-enhanced spectral mammography and the benefit of additional breast MRI," Journal of Cancer, vol. 6, no. 2, pp. 144-150, 2015.

[19] T. Shinjo and R. Kagami, "Radiological imaging change in a malignant bowel obstruction patient treated with octreotide," Supportive Care in Cancer, vol. 17, no. 6, pp. 753-755, 2009.

[20] K. P. Mcguire, E. S. Hwang, and A. Cantor, "Surgical patterns of care in patients with invasive breast cancer treated with neoadjuvant systemic therapy and breast magnetic resonance imaging: results of a secondary analysis of TBCRC 017," Annals of Surgical Oncology, vol. 22, no. 1, pp. 75-81, 2015.

[21] R. C. B. Aitken, "A growing edge of measurement of feelings [abridged]," Proceedings of the Royal Society of Medicine, vol. 62, no. 10, pp. 989-993, 1969.

[22] K. Taxis, B. Dean, and N. Barber, "The validation of an existing method of scoring the severity of medication administration errors for use in Germany [J]," Pharmacy World \& Science, vol. 24, no. 6, pp. 236-239, 2002.

[23] L. C. Loh, S. H. Puah, C. V. Ho et al., "Disability and breathlessness in asthmatic patients-a scoring method by repetitive inspiratory effort," Journal of Asthma Research, vol. 42, no. 10, pp. 853-858, 2005.

[24] A. T. Casey, J. M. Bland, and H. A. Crockard, "Development of a functional scoring system for rheumatoid arthritis patients with cervical myelopathy," Annals of the Rheumatic Diseases, vol. 55, no. 12, pp. 901-906, 1996.

[25] Q. Dan, M. A. Xiaolian, and O. Room, "The effect and influence of humanistic care in the whole process on the nursing of patients in the operating room," China Continuing Medical Education, vol. 46, no. 5, pp. 127-129, 2019.

[26] M. Nakabayashi, K. Teranishi, and M. Niikura, "A change in the degree of the mental health condition of mother with infants: the follow-up survey until an infant was 18 months from 4 months," Japanese journal of maternal health, vol. 46, pp. 655-665, 2006

[27] D. Jian-Ping, "Obstetrics and gynecology. Episiotomy postoperative nursing countermeasure analysis," World latest medicine Information, vol. 124, no. 5, pp. 46-49, 2016.

[28] Z. Tang, G. Zhao, and T. Ouyang, "Two-phase deep learning model for short-term wind direction forecasting," Renewable Energy, vol. 173, pp. 1005-1016, 2021. 